\title{
The Effect of Stimulus Rates in Chirp and Click Evoked Auditory Brainstem Response in Adults with Normal Hearing Sensitivity
}

https://doi.org/10.47210/bjohns.2020.v28i3.287

Susmi Pani, ${ }^{1}$ Archita Sahoo, ${ }^{1}$ Indranil Chatterjee, ${ }^{2}$ Palash Dutta ${ }^{2}$

\section{Introduction}

\section{ABSTRACT}

The effects of increasing stimulus repetition rate on the ABR using click stimuli have been investigated in normal and hearing impaired subjects with neurologic abnormality but there is limited study on the effect of stimulus repetition rate on ABR using chirp stimuli. The present study aims to compare the chirp evoked auditory brainstem responses with reference to changes in latency of peaks, interaural latency differences and interwave latency intervals as a function of rate and compare those responses with the click evoked auditory brainstem responses, in normal hearing subjects.

\section{Materials and Methods}

Total 30 normally hearing adults were considered for this study. All participants were screened for normal hearing sensitivity upto $8 \mathrm{kHz}$ in pure tone audiometry for middle ear pathology and central auditory processing disorder. Four parameters of $A B R$ were considered to assess in this study including absolute latency, interwave latency intervals, latency-rate function and interaural latency. ABR was done based on the protocol of this study.

\section{$\underline{\text { Results }}$}

Results revealed that there was a significant difference in the absolute latency and interwave intervals when the stimulus repetition rate was increased.

\section{Conclusion}

The latencies of wave III and V increases and waveform morphology changed as the stimulus repetition rate increased above 20/ sec. The absolute latency of wave III and V was found to be shorter than clicks and can be used especially in newborn hearing evaluation assuming in shorter time window.

$\underline{\text { Keywords }}$

Evoked Potentials, Auditory, Brain Stem; Chirp; Click; Latency; Stimulus Rate

$\mathrm{T}$ The auditory evoked potentials are the electrical responses of the nervous system to auditory stimuli. ${ }^{1}$ The ABR is a complex response to particular type of external stimuli that characterizes neural activity generated from the eighth cranial nerve

1 - Department Audiology and speech

pathology, AYJNISHD, Kolkata

2 - Lecturer in Audiology at AYJNISHD, Kolkata

Corresponding author:

Dr Susmi Pani

email: panisusmi@gmail.com i.e. vestibulocochlear nerve and neural centers and tracts within the brainstem which are responsive to auditory stimulation. Auditory brainstem response (ABR) audiometry was first described Jewett and Williston in $1971 .^{2}$

The ABR waveform revealed by summation usually has wave components (peaks and troughs) which are described by their amplitude and latency characteristics. By correlating the location of the lesion with the changes in the ABR, information about the origin of the different components of the ABR was obtained. ${ }^{3,4,5,6}$ The time interval between the stimulus onset and the peak of a 
waveform is referred as the absolute latency, more precisely, the absolute latency of a peak because it is related to the onset of the stimulus respect to the particular peak. The unit measurement of latency is millisecond (ms). Latency of ABR waveforms is the most consistent and robust characteristics and provides the nucleus of $\mathrm{ABR}$ interpretation and peak latencies should replicate within $0.1 \mathrm{~ms}$. In normal individuals, the absolute latency of wave I should occur approximately at $1.6 \mathrm{~ms}$ after stimulus onset, wave III at about $3.7 \mathrm{~ms}$ and wave $\mathrm{V}$ at about $5.6 \mathrm{~ms}$ for click presented at an intensity level of approximately $75 \mathrm{~dB}$ above normal threshold.

The limits of the normal latency range encompass either two or three standard deviations from the mean value. $^{7}$ Latency is affected by various factors; most commonly observed change is increase or decrease of stimulus intensity and repetition rate. Substantial maturational changes occur in the ABR during early life, both in terms of waveform morphology and latencies. Hecox and Galambos (1974) concluded that ABR waves in infants are incomplete, only wave I, III, $\mathrm{V}$ are observed, and interwave intervals are initially prolonged. ${ }^{8}$ At around 18 months of age other wave components emerge and wave III and V progressively shorten in latency. In infants wave I is more prominent than later waves because peripheral system matures before central auditory nervous system. ${ }^{9}$ The length of time window will vary with the age of the patients, the intensity and the type of the stimulus used. In adults a time window of $10-12 \mathrm{~ms}$ is sufficient, but testing below 18 months ABR components are delayed, therefore a time window of at least $15 \mathrm{~ms}$ is recommended. ${ }^{7}$ For eliciting a detectable ABR, the acoustic click is often thought to be an ideal stimulus because of its abrupt onset, a wide spectral spread, are inherent in transient signals; which elicit synchronous discharge from a large portion of cochlear fibers. ${ }^{10,11}$ For estimation of low frequency hearing status, frequency specific ABR using tone burst, which have a rapid rise time while still maintaining some frequency specificity, can be used. ${ }^{12}$

In creating a chirp stimulus, higher frequency components contributing to the stimulus are delayed in time relative to the lower frequency components. ${ }^{13}$ Through this stimulus generation, chirps are designed to offset cochlear delays and increase synchronous neural firing, resulting in increased response amplitude. ${ }^{14}$

\section{Materials and Methods}

Quasi Experimental design was used. There were 30 normal hearing participants, included 15 males and 15 females (mean age: 22.30 years, standard deviation: 2.10 years) within the age range $18-25$ years, with a thorough case history with normal medical and otologic history. All the subjects passed an otoscopic examination to ensure a normal external ear canal, had hearing sensitivity within normal limits $(<25 \mathrm{dBHL})$ at standard audiometric frequencies $(250 \mathrm{~Hz}$ to $8000 \mathrm{~Hz}$; ANSI 1996), having normal speech discrimination score was within the range of $80 \%-100 \%$ and normal middle ear function and tympanic membrane movement, as measured by tympanometry, no central auditory processing disorder and Auditory Neuropathy Spectrum Disorder.

Double sound treated room with permissible noise level no more than $25 \mathrm{~dB}$ as per ANSI S3.6- 2003was used. ABR instrument was calibrated as per manufacture specification. Subjects were asked to stay quiet and relaxed in order to avoid artifacts related to the muscles responses. After cleaning the surface of skin, electrodes were placed by the conductive electrode paste, attached to the skin with the tape. The electrode was placed at vertex or positive electrode $(\mathrm{Cz})$, two negative electrodes were placed on mastoid (A1 and A2) and the ground electrode was placed on forehead. The impedance was checked at beginning of the test and end of the test maintained at less than or equal to $5000 \mathrm{ohm}$, and the $2000 \mathrm{ohm}$ was the electrode impedance difference of each other. A total 2000 alternative stimulus was presented through insert earphones at the fixed level of $80 \mathrm{dBnHL}$. Filter setting was $100 \mathrm{~Hz}$ to $3000 \mathrm{~Hz}$ and the repetition rates were $11.4 / \mathrm{sec}, 20 / \mathrm{sec}, 27.1 / \mathrm{sec}, 27.7 /$ $\mathrm{sec}, 33.1 / \mathrm{sec} 44.1 / \mathrm{sec}$.

Only the ipsilateral recording was done. The latencies of each wave were plotted after acquisition of the data and absolute latency, interwave latency and interaural differences were measured.

Data was recorded for all the participants. The latency 
of peak I, III and V, interaural latency differences, interwave latency intervals was obtained at different stimulus rate at the fixed intensity level for comparing of two stimuli (chirp and click) in normal hearing adults. Data was processed in excel spread sheet, and analyzed using statistical package for the social Science software (Version 16.0). Descriptive analysis was used to calculate the normative. Independent-sample $t$ test, one-way Analysis of Variance, posthoc (Tukey) tests were performed to measure any significant differences in the latency value as a result of increasing stimulus rate.

\section{Results}

The objective of the present study was to develop normative data for chirp stimulus at six different stimulus repetition rates at a fixed intensity level $(80 \mathrm{dBnHL})$ for adult normal hearing population and to study the effect of stimulus rate on the ABR latencies and compare the auditory brainstem responses elicited by chirp stimulus and click stimulus in participants. Results were analyzed in terms of absolute latencies of waves I, III, V and interwave intervals of wave I-III, III-V and I-V, latency rate functions and interaural latency differences. (Table I)

Post hoc (Tukey) analysis for multiple comparison (Table II) showed significant mean difference in the absolute latency values of wave I between the stimulus repetition rates of $11.4 / \mathrm{sec}$ and $44.1 / \mathrm{sec}[\mathrm{p}=0.006]$ at $95 \%$ level of confidence. No significant mean differences have been found in corresponds to other mentioned rates i.e. $20 / \mathrm{sec}, 27.1 / \mathrm{sec}, 27.7 / \mathrm{sec}$ and $33.1 / \mathrm{sec}$. There was significance mean difference in the absolute latency of wave III between the stimulus repetition rates of $33.1 / \mathrm{sec}$ and $11.4 / \mathrm{sec}[\mathrm{p}=0.019]$, $44.1 / \mathrm{sec}$ and $20 / \mathrm{sec}[\mathrm{p}=0.001], 44.1 / \mathrm{sec}$ and $27.1 / \mathrm{sec}$ $[\mathrm{p}=0.001]$ and $44.1 / \mathrm{sec}$ and $27.7 / \mathrm{sec}[\mathrm{p}=0.027]$ and 44.1 and $11.4 / \mathrm{sec}[\mathrm{p}=0.000]$ at $95 \%$ level of confidence. No significant mean differences were found in corresponds to other mentioned rates; It was found that there was significant mean difference in the absolute latency of wave $\mathrm{V}$ between the stimulus repetition rates of 44.1/ $\mathrm{sec}$ and $33.1 / \mathrm{sec}[\mathrm{p}=0.044], 44.1 / \mathrm{sec}$ and $27.7 / \mathrm{sec}$ $[\mathrm{p}=0.000], 44.1 / \mathrm{sec}$ and $27.1 / \mathrm{sec}[\mathrm{p}=0.000], 44.1 / \mathrm{sec}$ and $20 / \mathrm{sec}[\mathrm{p}=0.000], 44.1 / \mathrm{sec}$ and $11.4 / \mathrm{sec}[\mathrm{p}=0.000]$,

Table I: Determine the normative values of absolute latency of wave I, III, V and interwave intervals of wave I-III, III-V and I-V at six different stimulation rates in chirp evoked ABR.

\begin{tabular}{|c|c|c|c|c|c|c|c|c|c|c|c|c|}
\hline \multirow{3}{*}{ WAVE } & \multicolumn{12}{|c|}{ REPETITION RATES (/SEC) } \\
\hline & \multicolumn{2}{|c|}{ 11.4/SEC } & \multicolumn{2}{|c|}{ 20/SEC } & \multicolumn{2}{|c|}{ 27.1/SEC } & \multicolumn{2}{|c|}{ 27.7/SEC } & \multicolumn{2}{|c|}{ 33.1/SEC } & \multicolumn{2}{|c|}{ 44.1/SEC } \\
\hline & MEAN & S.D & MEAN & S.D & MEAN & S.D & MEAN & S.D & MEAN & S.D & MEAN & S.D \\
\hline Wave I & 1.38 & 0.27 & 1.45 & 0.28 & 1.44 & 0.31 & 1.49 & 0.31 & 1.5 & 0.29 & 1.57 & 0.25 \\
\hline Wave III & 2.99 & 0.4 & 3.06 & 0.42 & 3.05 & 0.44 & 3.12 & 0.36 & 3.22 & 0.34 & 3.34 & 0.35 \\
\hline Wave V & 4.3 & 0.42 & 4.39 & 0.46 & 4.51 & 0.54 & 4.54 & 0.47 & 4.69 & 0.48 & 4.95 & 0.48 \\
\hline $\begin{array}{l}\text { Interwave } \\
\text { interval of } \\
\text { Wave I-III }\end{array}$ & 1.6 & 0.33 & 1.59 & 0.37 & 1.61 & 0.37 & 1.62 & 0.31 & 1.74 & 0.31 & 1.76 & 0.34 \\
\hline $\begin{array}{c}\text { Interwave } \\
\text { interval } \\
\text { of Wave } \\
\text { III-V }\end{array}$ & 1.3 & 0.35 & 1.33 & 0.35 & 1.46 & 0.36 & 1.43 & 0.39 & 1.46 & 0.33 & 1.62 & 0.32 \\
\hline $\begin{array}{l}\text { Interwave } \\
\text { interval of } \\
\text { Wave I-V }\end{array}$ & 2.91 & 0.41 & 2.94 & 0.43 & 3.07 & 0.54 & 3.06 & 0.42 & 3.19 & 0.39 & 3.38 & 0.46 \\
\hline
\end{tabular}


Table II: One-Way ANOVA showing the effect of stimulus repetition rates on absolute latency values of waves in normal hearing adults.

\begin{tabular}{|c|c|c|c|c|c|c|c|c|c|c|}
\hline \multirow{3}{*}{ WAVE } & \multicolumn{10}{|c|}{ LATENCY VALUES } \\
\hline & \multicolumn{5}{|c|}{ BETWEEN GROUPS } & \multicolumn{3}{|c|}{ WITHIN GROUPS } & \multicolumn{2}{|c|}{ TOTAL } \\
\hline & $\begin{array}{l}\text { SUM OF } \\
\text { SQUARE }\end{array}$ & DF & MEAN & $\mathbf{F}$ & SIG. & $\begin{array}{l}\text { SUM OF } \\
\text { SQUARE }\end{array}$ & DF & MEAN & $\begin{array}{c}\text { SUM OF } \\
\text { SQUARES }\end{array}$ & DF \\
\hline Wave I & 1.217 & 5 & 0.243 & 2.856 & 0.015 & 30.158 & 354 & 0.085 & 31.375 & 359 \\
\hline Wave III & 4.875 & 5 & 0.975 & 6.351 & $\mathbf{0}$ & 54.346 & 354 & 0.154 & 59.222 & 359 \\
\hline Wave V & 15.801 & 5 & 3.16 & 13.69 & $\mathbf{0}$ & 81.72 & 354 & 0.231 & 97.521 & 359 \\
\hline $\begin{array}{l}\text { Interwave } \\
\text { intervals of } \\
\text { Wave I-III }\end{array}$ & 1.669 & 5 & 0.334 & 2.811 & 0.017 & 42.035 & 354 & 0.119 & 43.704 & 359 \\
\hline $\begin{array}{l}\text { Interwave } \\
\text { intervals of } \\
\text { Wave III- V }\end{array}$ & 378.829 & 5 & 75.766 & 1.188 & 0.315 & 22576.678 & 354 & 63.776 & 22955.506 & 359 \\
\hline $\begin{array}{l}\text { Interwave } \\
\text { intervals of } \\
\text { Wave I-V }\end{array}$ & 9.047 & 5 & 1.809 & 9.322 & $\mathbf{0}$ & 68.707 & 354 & 0.194 & 77.753 & 359 \\
\hline
\end{tabular}

$33.1 / \mathrm{sec}$ and $20 / \mathrm{sec}[\mathrm{p}=0.010]$, and $33.1 / \mathrm{sec}$ and $11.4 /$ sec $[p=0.000]$ at $95 \%$ level of confidence. No significant mean differences were found in corresponds to other mentioned rates; in case of interwave intervals, there is no significant mean difference in the interwave intervals of wave I-III and III-V between the all selected stimulus repetition rates. But there is positively significance mean difference in the interwave interval of wave I-V between the stimulus repetition rates of $44.1 / \mathrm{sec}$ and $11.4 / \mathrm{sec}$ $[\mathrm{p}=0.000], 44.1 / \mathrm{sec}$ and $20 / \mathrm{sec}[\mathrm{p}=0.000], 44.1 / \mathrm{sec}$ and $27.1 / \sec [p=0.002], 44.1 / \sec$ and $27.1 / \sec [p=0.001]$, $33.1 / \mathrm{sec}$ and $20 / \mathrm{sec}[\mathrm{p}=0.21]$, and $33.1 / \mathrm{sec}$ and $11.4 / \mathrm{sec}$ $[p=0.009]$ at $95 \%$ level of confidence. No significant mean differences were found in corresponds to other mentioned rates. The mean difference is significant at the 0.05 level.

Regarding interaural latency differences, through $t$ test statistics $(p>0.05)$ indicated that there was no significant difference in absolute latency of wave I, III, V, interwave interval of wave I-III, III-V and I-V latencies at six selected repetition rates.

By calculating $t$ test statistics $(p>0.05)$ There was no significant difference in absolute latency of wave I between chirp and click evoked responses for six stimulus repetition rates but there was significant difference in absolute latency of wave III, V, interwave interval of wave I-III, interwave intervals of wave III-V, and interwave intervals of wave I-V between chirp and click ABR at six selected repetition rates.

\section{Discussion}

Present study aims to find out the normative value of absolute latency of ABR waves and the interwave intervals for chirp evoked ABR at different stimulus repetition rates and to find out the effect of repetition rates on the ABR waves when it is evoked by chirp stimulus and to compare the responses elicited by chirp stimulus and click stimulus in normal hearing participants.

Interwave interval of I-III, III-V, I-V. (Fig. 1) There was a significant difference in the absolute latency value of wave I, III, V as the effect of stimulus repetition rate for chirp evoked ABR i.e. absolute latency was prolonged as the repetition rate was increased. No significant differences were observed for interwave intervals of wave III-V as an effect of increasing stimulus repetition rate and for interwave intervals of wave I-III, I-V was observed for chirp evoked ABR. 


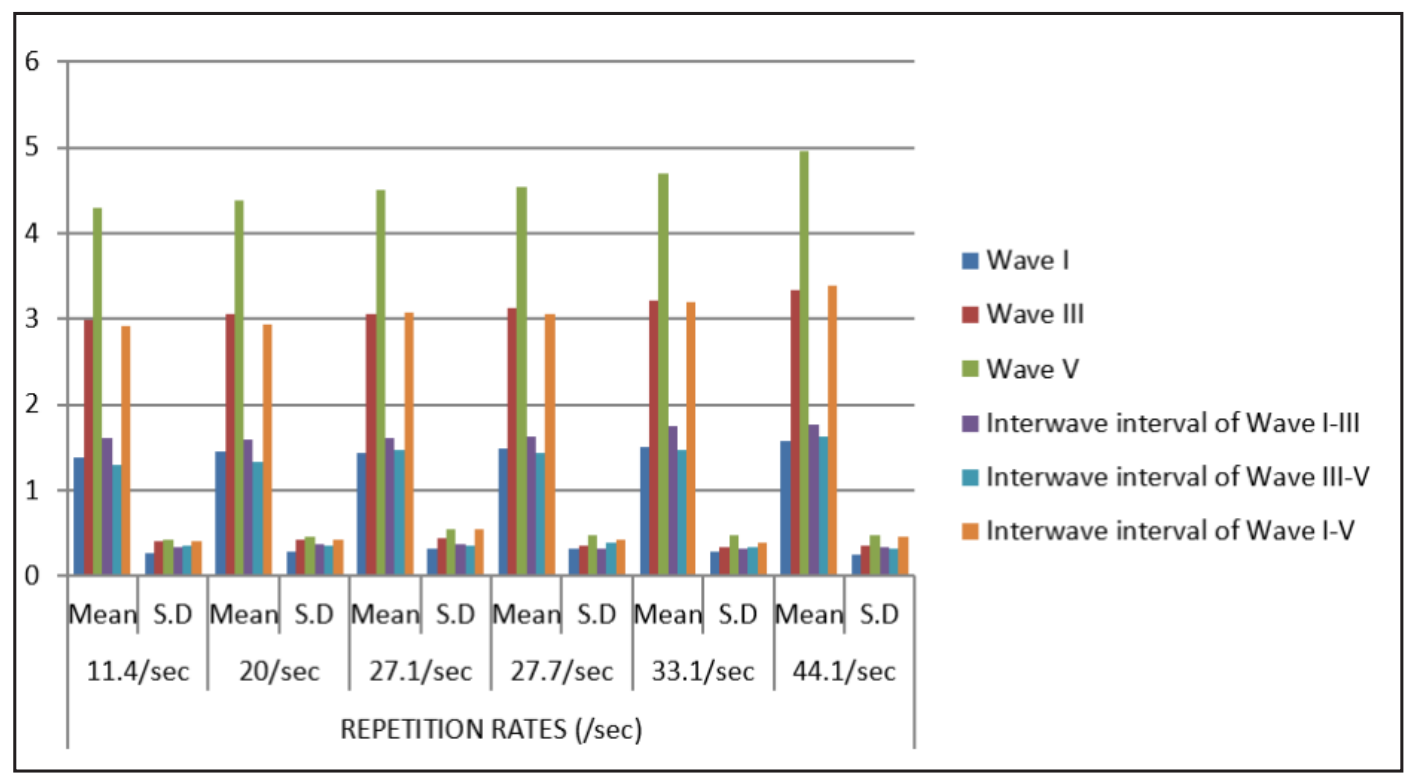

Fig. 1. Determine the normative values of absolute latency of wave I, III, V and interwave intervals of wave I-III, III-V and I-V at six different stimulation rates in chirp evoked ABR.

The present study showed that there was no significant inter-aural difference in absolute latency of wave I, wave III, wave $\mathrm{V}$ and interwave intervals of wave I-III, III-V and $\mathrm{I}-\mathrm{V}$ in normal hearing adults at different stimulus repetition rates in chirp evoked $\mathrm{ABR}$.

There was no significant difference in absolute latency of wave I between two stimuli i.e. clicks and CE-Chirp, but the difference in the absolute latency

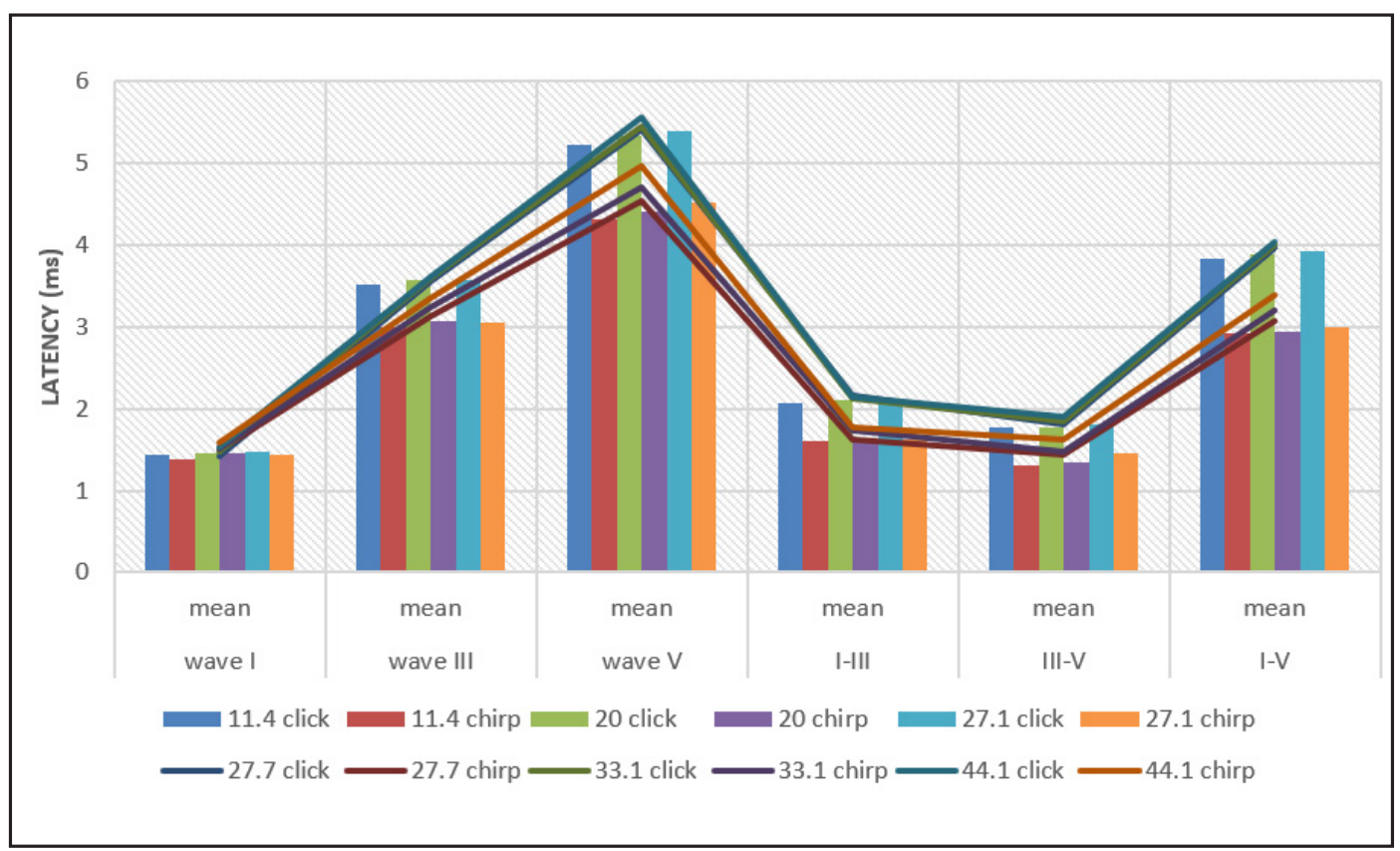

Fig. 2. Chart showing the mean difference of the absolute latency of wave I, III, V and inter-wave intervals of I-III, III-V, I-Vin six different stimulus rates between chirp-evoked ABR and click-evoked ABR in normal hearing adults. 
of wave III and wave $\mathrm{V}$ was significant between two stimuli i.e. the absolute latency value of wave III and Vis more for click stimulus than chirp stimulusat all stimulus repetition rates. (Fig. 2) Present study showed that the difference in the interwave intervals of wave I-III, III-V,I-V was significant between two stimuli i.e. clicks and CE-Chirp.

The findings of the present study were supported by the findings of Cebulla et al that Chirp-evoked responses showed clearly larger wave amplitudes and shorter wave's latencies compared to those evoked by click. ${ }^{15}$

The present study also supported by the findings of Hamada et al that statistically significant difference of wave V latency when comparing between click, low frequency and high frequency chirp in all test conditions in normal hearing subjects. ${ }^{16}$

This study supports the finding of Hall (2016) that the amplitude for the ABR is usually up to two times larger for chirp stimuli than for traditional stimuli. ${ }^{17}$ That means one can identify wave $\mathrm{V}$ with more confidence, which leads to reduce test time than clicked evoked ABR.

The possible explanation for shift in latency of ABR waves as a function of rate in chirp stimuli is due to cumulative neural fatigue and adaptation and incomplete recovery involving hair-cell-cochlear nerve junction and also subsequent synaptic transmission. ${ }^{18}$ The difference in the latency value of ABR component for chirp and clicks can be explained by the simultaneous depolarization of all frequency regions of the basilar membrane. Chirps are designed to offset cochlear delays and increase synchronous neural firing. ${ }^{14}$

\section{Conclusion}

As stimulus repetition rate increases above $20 / \mathrm{sec}$, the latencies of wave III and V increases and waveform morphology changes. The absolute latency of wave III and $\mathrm{V}$ was found to be shorter than clicks and can be used especially in newborn hearing evaluation assuming in shorter time window, but further study may need for establishment of normative in newborn, and comparison of stimulus rate in disorder population at various age groups, so that chirp evoked ABR can be used in diagnostic purpose. The chirp ABR helps in assessing hearing thresholds.

\section{Limitations of the Study}

I. As compared to the click evoked ABR the recommended stimulus rate in chirp evoked ABR was not computed.

II. Stimulus repetition rate beyond $44.1 / \mathrm{sec}$ could not be analyzed due to instrumental limitation.

\section{Future Directions}

I. Comparison of stimulus repetition rates in chirp ABR in newborn population.

II. Comparison of stimulus repetition rates in tone burst and chirp stimuli.

III. To compare the chirp stimulus in diseased population.

IV. To compare the chirp stimulus in male and female.

\section{References}

1. Stapells DR, Picton TW, Abalo MP, Read D, Smith A. Frequency specificity in evoked potential audiometry. In T.T. Jacobson (Ed) The auditory brainstem response. San Diego: College Hill Press, Inc. 1985; 147-77

2. Jewett D, Williston J. Auditory-evoked far fields averaged from the scalp of humans. Brain 1971;94(4):681-96

3. Sohmer H, Feinmesser M, Szabo G. Sources of electrocochleographic responses as studied in patients with brain damage. Electroencephalography and Clinical Neurophysiology 1974; 37(6):663-9

4. Starr A, Achor L. Auditory Brain Stem Responses in Neurological Disease. Archives of Neurology. 1975; 32(11):761-8

5. Starr A, Hamilton AE. Correlation between confirmed sites of neurological lesions and abnormalities of far-field auditory brainstem responses. Electroencephalography and Clinical Neurophysiology 1976;41(6):595-608

6. Stockard J, Rossiter V. Clinical and pathologic correlates of brain stem auditory response abnormalities. Neurology 1977; 27(4):316

7. Hood LJ. Clinical applications of the Auditory Brainstem Response. San Deigo: Singular Publishing Group, Inc. 1998; 49-63

8. Hecox K, Galambos R. Brain Stem Auditory Evoked Responses in Human Infants and Adults. Archives of Otolaryngology Head and Neck Surgery 1974;99(1):30-33 
9. Stockard J, Westmoreland B. Technical Considerations in the Recording and Interpretation of the Brainstem Auditory Evoked Potential for Neonatal Neurologic Diagnosis. American Journal of EEG Technology 1981; 21(1):31-54

10. Kodera K, Yamane H, Yamada O, Suzuki J. The Effect of Onset, Offset and Rise-Decay Times of Tone Bursts on Brain Stem Response. Scandinavian Audiology 1977; 6(4):205-10

11. Gorga M, Thornton A. The Choice of Stimuli for ABR Measurements. Ear and Hearing. 1989; 10(4):217-30

12. Picton TW, Ouelette K, Hamel G, Smith AD. Brainstem evoked potential to tone pips in notched noise. Journal of Otolaryngology 1979; 8:289-314

13. Dau T, Wegner O, Mellert V, Kollmeier B. Auditory brainstem responses with optimized chirp signals compensating basilarmembrane dispersion. The Journal of the Acoustical Society of America. 2000;107(3):1530-40

14. Fobel O, Dau T. Searching for the optimal stimulus eliciting auditory brainstem responses in humans. The Journal of the Acoustical Society of America 2004;116(4):2213-22

15. Cebulla M, Lurz H, Shehata-Dieler W. Evaluation of waveform, latency and amplitude values of chirp ABR in newborns. International Journal of Pediatric Otorhinolaryngology. 2014; 78(4):631-6

16. Hamada SM, Latif SMA, Abumoussa HI. The Verification of ABR Response by Using the Chirp Stimulus in Moderate Sensorineural Hearing Loss. Medical Journal of Cairo University 2013:81(2), 21-6

17. Hall JW III. Update on auditory evoked responses: value of chirp stimuli in ABR/ASSR measurement. Audiology Online; June 27, 2016; article 17434.

18. Teas D, Eldredge D, Davis H. Cochlear Responses to Acoustic Transients: An Interpretation of Whole-Nerve Action Potentials. The Journal of the Acoustical Society of America 1962; 34(9B):1438-59. 